\title{
The Benjamin-Bona-Mahony equation with dissipative memory
}

\author{
Filippo Dell'Oro, Youcef Mammeri and Vittorino Pata
}

\begin{abstract}
We show that the nonlinear contraction semigroup generated by the Benjamin-Bona-Mahony equation with dissipative memory

$$
u_{t}-u_{t x x}+u_{x}-\int_{0}^{\infty} g(s) u_{x x}(t-s) \mathrm{d} s+u^{p} u_{x}=0
$$
\end{abstract}

is exponentially stable for every $p \in \mathbb{N}$.

Mathematics Subject Classification. 35B35, 35B40, 35F25, 45K05.

Keywords. Benjamin-Bona-Mahony equation, Nonlinear contraction semigroup, Dissipative memory, Exponential stability.

\section{Introduction}

This paper deals with the propagation of the one-directional small amplitude long waves in shallow water. In the conservative context, such waves are described by the Korteweg-de Vries (KdV) equation [19]

$$
u_{t}+u_{x x x}+u_{x}+u u_{x}=0
$$

where $u=u(x, t): I \times \mathbb{R}^{+} \rightarrow \mathbb{R}$ denotes the wave surface, $I \subset \mathbb{R}$ being a bounded interval. In 1972, Benjamin, Bona and Mahony [4] proposed to replace the term $u_{x x x}$ by $-u_{t x x}$, thus obtaining the regularized $\mathrm{KdV}$ equation (here called BBM equation)

$$
u_{t}-u_{t x x}+u_{x}+u u_{x}=0 .
$$

The equation above can be directly derived from Newton's second law, in the same way the KdV equation is obtained from the Euler one [21,22]. In the dissipative context, namely, in the modeling of long gravity waves when also viscosity is taken into account (see e.g. $[6,10,18]$ ), the BBM equation turns into

$$
u_{t}-u_{t x x}-\nu u_{x x}+u_{x}+u u_{x}=0, \quad \nu>0,
$$


or, more generally,

$$
u_{t}-u_{t x x}-\nu u_{x x}+(f(u))_{x}=q
$$

where $f$ and $q$ are a suitable nonlinear function and a time-independent forcing term, respectively. Actually, it is a standard matter to prove that the initial value problem associated to (1.2) with the Dirichlet boundary condition is globally well-posed in the Sobolev space $H_{0}^{1}(I)$. Hence, it generates a nonlinear solution semigroup $S(t)$ on $H_{0}^{1}(I)$ defined by the action

$$
u_{0} \mapsto S(t) u_{0}=u(t),
$$

where $u(t)$ is the unique solution at time $t$ with initial datum $u_{0} \in H_{0}^{1}(I)$. Concerning the longtime dynamics, Wang and Yang [29,31] proved the existence of a finite-dimensional global attractor for $S(t)$. Since the semigroup is not compact in $H_{0}^{1}(I)$, the proof is based on the weak continuity of $S(t)$ and energy methods inspired by Ghidaglia's work (see e.g. [12,27,28]). Other results can be found for instance in $[3,5,15,20,27,30]$ and references therein.

Coming back to the homogeneous model (1.1), multiplying in $L^{2}(I)$ the equation by $2 u$ and exploiting the Dirichlet boundary condition, the (twice) energy

$$
E(t)=\left\|S(t) u_{0}\right\|_{H^{1}(I)}^{2}
$$

is readily seen to satisfy the equality

$$
\frac{\mathrm{d}}{\mathrm{d} t} E(t)=-2 \nu\left\|u_{x}(t)\right\|_{L^{2}(I)}^{2} .
$$

Hence, in light of the Poincaré inequality and the Gronwall lemma, we deduce the exponential stability

$$
E(t) \leq E(0) \mathrm{e}^{-\kappa t},
$$

where $\kappa$ is a strictly positive constant depending only on $\nu$ and the interval $I$. Note that, in the conservative limit case $\nu=0$, the energy is preserved, namely $E(t)=E(0)$. Many other papers related with damped BBM equations with weaker dissipation are nowadays present in the literature (see $[1,2,7,17]$ ). Still, to the best of our knowledge, none of them is dealing with dispersive equations with dissipative memory.

Motivated by the discussion above, our aim is to study the asymptotic behavior of the integro-differential equation

$$
u_{t}-u_{t x x}+u_{x}-\int_{0}^{\infty} g(s) u_{x x}(t-s) \mathrm{d} s+u^{p} u_{x}=0
$$

in the unknown $u=u(x, t): I \times \mathbb{R} \rightarrow \mathbb{R}$, complemented with the Dirichlet boundary condition

$$
u_{\mid \partial I}=0 .
$$

Here $p \in \mathbb{N}$ is a fixed constant (when $p=0$ the model becomes linear), while $g$ is a bounded convex summable function on $[0, \infty)$ of total mass

$$
\int_{0}^{\infty} g(s) \mathrm{d} s=1
$$


having the explicit form

$$
g(s)=\int_{s}^{\infty} \mu(y) \mathrm{d} y,
$$

where $\mu: \mathbb{R}^{+}=(0, \infty) \rightarrow[0, \infty)$, the so-called memory kernel, is a nonincreasing absolutely continuous summable function of total mass

$$
\varkappa:=\int_{0}^{\infty} \mu(s) \mathrm{d} s=g(0)>0 .
$$

Moreover, the function $u$ is supposed to be known for all $t \leq 0$. From the physical viewpoint, Eq. (1.3) can be interpreted as a memory relaxation of the dissipative BBM model (1.1) which, setting $\nu=1$, is formally recovered when $p=1$ and the kernel $g$ collapses into the Dirac mass at zero. It is also worth noting that the memory term provides a more realistic description of the Fick's law. In particular, it prevents the infinite propagation speed of regularization $[9,26]$. In our opinion, this motivates and justifies the interest in studying the asymptotic properties of such a model, as compared to the "classical" dissipative case discussed above. In this work we prove that the nonlinear solution semigroup generated by (1.3), acting on a suitable Hilbert space accounting for the presence of the memory, remains exponentially stable.

In order to explain the mathematical difficulties encountered in the analysis, we begin to observe that, also at a linear level, the exponential stability of (1.3) is much harder to prove than the one of (1.1). An enlightening example is provided by a comparison between the classical heat equation

$$
u_{t}-u_{x x}=0
$$

with the Dirichlet boundary condition and its memory relaxation, i.e. the Gurtin-Pipkin equation [16]

$$
u_{t}-\int_{0}^{\infty} g(s) u_{x x}(t-s) \mathrm{d} s=0 .
$$

In the first case, similarly to (1.1), the exponential stability is almost trivial, whereas the exponential stability of the Gurtin-Pipkin model has been proved only in recent years [13]. In the nonlinear situation the picture is even worse. Indeed, although the asymptotic analysis of the one-dimensional reactiondiffusion equation is carried out under quite general assumptions, the corresponding nonlinear Gurtin-Pipkin case suffers from serious drawbacks, and requires the choice of specific memory kernels concentrated at zero [14]. For the BBM equation the scenario is similar: while adding a further nonlinearity $h(u)$ in (1.2) does not cause any essential extra difficulty, the picture becomes much more involved when dissipative memory is introduced. In particular, even showing exponential stability in the homogeneous case (as we do in the present paper) is not at all an easy task. Concerning the existence of the global attractor when further nonlinearities and/or source terms are present, the techniques devised in this work do not apply and, at the moment, an answer seems out of reach. 
Plan of the paper. In the next Sect. 2 we introduce the functional setting and the notation, while in Sect. 3 we establish the existence of the solution semigroup. The final Sects. 4 and 5 are devoted to the main result about exponential stability.

\section{Functional setting and notation}

In what follows, $\langle\cdot, \cdot\rangle$ and $\|\cdot\|$ will denote the standard inner product and norm on the Hilbert space $L^{2}(I)$. In order to simplify the calculation, we introduce the strictly positive operators

$$
A=-\partial_{x x} \quad \text { with } \quad \mathfrak{D}(A)=H^{2}(I) \cap H_{0}^{1}(I) \Subset L^{2}(I)
$$

and

$$
B=I+A \quad \text { with } \quad \mathfrak{D}(B)=\mathfrak{D}(A) .
$$

The operator $B$ commutes with $A$ and the bilinear form

$$
\langle u, v\rangle_{1}=\left\langle B^{\frac{1}{2}} u, B^{\frac{1}{2}} v\right\rangle
$$

defines an equivalent inner product on the space $H_{0}^{1}(I)$ with induced norm

$$
\|u\|_{1}^{2}=\|u\|^{2}+\left\|u_{x}\right\|^{2}
$$

and we have the Poincaré inequality

$$
\frac{\lambda_{1}}{1+\lambda_{1}}\|u\|_{1}^{2} \leq\left\|u_{x}\right\|^{2}
$$

where $\lambda_{1}>0$ is the first eigenvalue of $A$. Finally, we consider the so-called memory space

$$
\mathcal{M}=L_{\mu}^{2}\left(\mathbb{R}^{+} ; H_{0}^{1}(I)\right)
$$

of square summable $H_{0}^{1}$-valued functions on $\mathbb{R}^{+}$with respect to the measure $\mu(s) \mathrm{d} s$, endowed with the inner product

$$
\langle\eta, \xi\rangle_{\mathcal{M}}=\int_{0}^{\infty} \mu(s)\left\langle\eta_{x}(s), \xi_{x}(s)\right\rangle \mathrm{d} s .
$$

The infinitesimal generator of the right-translation semigroup on $\mathcal{M}$ is the linear operator

$$
T \eta=-\eta^{\prime}
$$

with domain

$$
\mathfrak{D}(T)=\left\{\eta \in \mathcal{M}: \eta^{\prime} \in \mathcal{M}, \lim _{s \rightarrow 0}\left\|\eta_{x}(s)\right\|=0\right\},
$$

the prime standing for the weak derivative with respect to the internal variable $s \in \mathbb{R}^{+}$. Defining the nonnegative functional

$$
\Gamma[\eta]=-\int_{0}^{\infty} \mu^{\prime}(s)\left\|\eta_{x}(s)\right\|^{2} \mathrm{~d} s,
$$

an integration by parts together with a limiting argument yield the equality (see $[8,24])$

$$
2\langle T \eta, \eta\rangle_{\mathcal{M}}=-\Gamma[\eta] .
$$


The phase space of our problem will be

$$
\mathcal{H}=H_{0}^{1}(I) \times \mathcal{M}
$$

endowed with the norm

$$
\|(u, \eta)\|_{\mathcal{H}}^{2}=\|u\|_{1}^{2}+\|\eta\|_{\mathcal{M}}^{2}
$$

\section{The contraction semigroup}

We translate the problem in the so-called history space framework of Dafermos [9]. To this aim, introducing the auxiliary variable

$$
\eta=\eta^{t}(x, s)=\int_{0}^{s} u(x, t-y) \mathrm{d} y,
$$

accounting for the integrated past history of $u$, we rewrite (1.3) as

$$
\begin{aligned}
& B u_{t}+u_{x}+\int_{0}^{\infty} \mu(s) A \eta(s) \mathrm{d} s+u^{p} u_{x}=0, \\
& \eta_{t}=T \eta+u
\end{aligned}
$$

By means of standard arguments based on a Galerkin approximation procedure, one can show that system (3.1)-(3.2) above is well-posed in the phase space $\mathcal{H}$. In particular, the solution continuously depends on the initial data. As a consequence, it generates a strongly continuous solution semigroup

defined by the action

$$
S(t): \mathcal{H} \rightarrow \mathcal{H}
$$

where

$$
z_{0}=\left(u_{0}, \eta_{0}\right) \mapsto S(t) z_{0}=z(t),
$$

$$
z(t)=\left(u(t), \eta^{t}\right)
$$

is the unique (weak) solution to (3.1)-(3.2) with initial datum $z(0)=z_{0}$. Introducing (twice) the energy at time $t \geq 0$ corresponding to the initial datum $z_{0} \in \mathcal{H}$ as

$$
E(t)=\left\|S(t) z_{0}\right\|_{\mathcal{H}}^{2},
$$

we multiply (3.1) by $2 u$ in $L^{2}(I)$ and (3.2) by $2 \eta$ in $\mathcal{M}$. Summing up, we obtain

$$
\frac{\mathrm{d}}{\mathrm{d} t} E+2\left\langle u_{x}, u\right\rangle+2\left\langle u^{p} u_{x}, u\right\rangle=2\langle T \eta, \eta\rangle_{\mathcal{M}}
$$

Since, due to the boundary condition,

$$
2\left\langle u_{x}, u\right\rangle+2\left\langle u^{p} u_{x}, u\right\rangle=\int_{I} \frac{\mathrm{d}}{\mathrm{d} x}\left(u^{2}(x)\right) \mathrm{d} x+\frac{2}{p+2} \int_{I} \frac{\mathrm{d}}{\mathrm{d} x}\left(u^{p+2}(x)\right) \mathrm{d} x=0,
$$

an exploitation of (2.2) provides the energy identity

$$
\frac{\mathrm{d}}{\mathrm{d} t} E+\Gamma[\eta]=0
$$

In particular, since the functional $\Gamma[\eta]$ is nonnegative, we have the control

$$
E(t) \leq E(0)
$$

meaning that $S(t)$ is actually a contraction semigroup. 


\section{Exponential stability}

For the longterm analysis, the memory kernel $\mu$ is supposed to satisfy the additional assumption (see [9])

$$
\mu^{\prime}(s)+\delta \mu(s) \leq 0
$$

for some $\delta>0$ and almost every $s \in \mathbb{R}^{+}$. Note that $\mu$ can be unbounded in a neighborhood of zero.

The main result of the paper reads as follows.

Theorem 4.1. There exist a strictly positive constant $\omega$, depending on $\mu$ and the length of the interval $|I|$, and an increasing positive function $\mathcal{Q}_{p}$, depending besides on $\mu$ and $|I|$ also on $p$, such that

$$
E(t) \leq \mathcal{Q}_{p}(E(0)) \mathrm{e}^{-\omega t}
$$

In order to prove Theorem 4.1, we need to introduce an auxiliary energytype functional. First, due to the possible singularity of $\mu$ at zero, we choose $s_{\star}>0$ such that

$$
\int_{0}^{s_{\star}} \mu(s) \mathrm{d} s \leq \frac{\varkappa}{4}
$$

Then, defining the truncated kernel

$$
\rho(s)=\mu\left(s_{\star}\right) \chi_{\left(0, s_{\star}\right]}(s)+\mu(s) \chi_{\left(s_{\star}, \infty\right)}(s),
$$

for $\varepsilon>0$ we consider the functional

$$
\Psi_{\varepsilon}(t)=-\varepsilon \int_{0}^{\infty} \rho(s)\left\langle u_{x}(t), \eta_{x}^{t}(s)\right\rangle \mathrm{d} s .
$$

Since $\rho(s) \leq \mu(s)$, it is easily seen that

$$
\left|\Psi_{\varepsilon}(t)\right| \leq \alpha \varepsilon E(t)
$$

for every $t \geq 0$, for some universal constant $\alpha=\alpha(\mu,|I|)>0$.

Lemma 4.2. There exist universal constants $\beta, \gamma>0$, depending only on $\mu$ and $|I|$ but independent on $p$ and the initial energy $E(0)$, such that the inequality

$$
\frac{\mathrm{d}}{\mathrm{d} t} \Psi_{\varepsilon}(t)+\frac{\varepsilon \varkappa}{4}\left\|u_{x}(t)\right\|^{2} \leq \frac{\delta}{4}\left\|\eta^{t}\right\|_{\mathcal{M}}^{2}+\beta \varepsilon \Gamma\left[\eta^{t}\right]
$$

holds for every $t \geq 0$, whenever $\varepsilon E(0)^{p} \leq \gamma$.

Proof. In what follows $C \geq 0$ will denote a generic constant possibly depending on the structural quantities of the problem but independent on $p$ and the initial 
energy $E(0)$. We compute the time derivative of $\Psi_{\varepsilon}$ as

$$
\begin{aligned}
\frac{\mathrm{d}}{\mathrm{d} t} \Psi_{\varepsilon}= & -\varepsilon \int_{0}^{\infty} \rho(s)\left\langle u_{t x}, \eta_{x}(s)\right\rangle \mathrm{d} s-\varepsilon \int_{0}^{\infty} \rho(s)\left\langle u_{x}, \eta_{t x}(s)\right\rangle \mathrm{d} s \\
= & \varepsilon \int_{0}^{\infty} \rho(s)\left\langle B^{-1} u_{x}, A \eta(s)\right\rangle \mathrm{d} s \\
& +\varepsilon \int_{0}^{\infty} \rho(s)\left(\int_{0}^{\infty} \mu(\sigma)\left\langle B^{-1} A \eta(\sigma), A \eta(s)\right\rangle \mathrm{d} \sigma\right) \mathrm{d} s \\
& +\varepsilon \int_{0}^{\infty} \rho(s)\left\langle B^{-1}\left(u^{p} u_{x}\right), A \eta(s)\right\rangle \mathrm{d} s-\varepsilon \int_{0}^{\infty} \rho(s)\left\langle u_{x}, T \eta_{x}(s)\right\rangle \mathrm{d} s \\
& -\varepsilon\left\|u_{x}\right\|^{2} \int_{0}^{\infty} \rho(s) \mathrm{d} s .
\end{aligned}
$$

Then appealing to (4.1) we estimate

$$
\begin{aligned}
& \varepsilon \int_{0}^{\infty} \rho(s)\left\langle B^{-1} u_{x}, A \eta(s)\right\rangle \mathrm{d} s+\varepsilon \int_{0}^{\infty} \rho(s)\left(\int_{0}^{\infty} \mu(\sigma)\left\langle B^{-1} A \eta(\sigma), A \eta(s)\right\rangle \mathrm{d} \sigma\right) \mathrm{d} s \\
& \quad \leq C \varepsilon\left(\left\|u_{x}\right\|\|\eta\|_{\mathcal{M}}+\|\eta\|_{\mathcal{M}}^{2}\right) \\
& \quad \leq \frac{\varkappa \varepsilon}{8}\left\|u_{x}\right\|^{2}+C \varepsilon\|\eta\|_{\mathcal{M}}^{2} \\
& \quad \leq \frac{\varkappa \varepsilon}{8}\left\|u_{x}\right\|^{2}+C \varepsilon \Gamma[\eta] .
\end{aligned}
$$

Moreover, using (4.2) and the equality $\rho(s)=\mu(s)$ for $s \geq s_{\star}$, we have

$$
-\varepsilon\left\|u_{x}\right\|^{2} \int_{0}^{\infty} \rho(s) \mathrm{d} s \leq-\varepsilon\left\|u_{x}\right\|^{2} \int_{s_{\star}}^{\infty} \mu(s) \mathrm{d} s \leq-\frac{3 \varkappa \varepsilon}{4}\left\|u_{x}\right\|^{2} .
$$

Integrating by parts in $s$, we infer that

$$
\begin{aligned}
-\varepsilon \int_{0}^{\infty} \rho(s)\left\langle u_{x}, T \eta_{x}(s)\right\rangle \mathrm{d} s & =-\varepsilon \int_{s_{\star}}^{\infty} \mu^{\prime}(s)\left\langle u_{x}, \eta_{x}(s)\right\rangle \mathrm{d} s \\
& \leq \varepsilon\left\|u_{x}\right\|\left(-\int_{s_{\star}}^{\infty} \mu^{\prime}(s)\left\|\eta_{x}(s)\right\| \mathrm{d} s\right) \\
& \leq \frac{\varkappa \varepsilon}{8}\left\|u_{x}\right\|^{2}+C \varepsilon\left(-\int_{s_{\star}}^{\infty} \mu^{\prime}(s)\left\|\eta_{x}(s)\right\| \mathrm{d} s\right)^{2} .
\end{aligned}
$$

Therefore, since

$$
\left(-\int_{s_{\star}}^{\infty} \mu^{\prime}(s)\left\|\eta_{x}(s)\right\| \mathrm{d} s\right)^{2} \leq \int_{s_{\star}}^{\infty} \mu^{\prime}(s) \mathrm{d} s \int_{s_{\star}}^{\infty} \mu^{\prime}(s)\left\|\eta_{x}(s)\right\|^{2} \mathrm{~d} s \leq \mu\left(s_{\star}\right) \Gamma[\eta],
$$

we obtain

$$
-\varepsilon \int_{0}^{\infty} \rho(s)\left\langle u_{x}, T \eta_{x}(s)\right\rangle \mathrm{d} s \leq \frac{\varkappa \varepsilon}{8}\left\|u_{x}\right\|^{2}+C \varepsilon \Gamma[\eta]
$$


Finally, exploiting the embedding $H^{1}(I) \subset L^{\infty}(I)$ and (3.4),

$$
\begin{aligned}
\varepsilon \int_{0}^{\infty} \rho(s)\left\langle B^{-1}\left(u^{p} u_{x}\right), A \eta(s)\right\rangle \mathrm{d} s & \leq C \varepsilon\left\|u^{p} u_{x}\right\|\|\eta\|_{\mathcal{M}} \leq C \varepsilon\|u\|_{L^{\infty}}^{p}\left\|u_{x}\right\|\|\eta\|_{\mathcal{M}} \\
& \leq C \varepsilon\|u\|_{1}^{p}\left\|u_{x}\right\|\|\eta\|_{\mathcal{M}} \leq C \varepsilon E(0)^{\frac{p}{2}}\left\|u_{x}\right\|\|\eta\|_{\mathcal{M}} \\
& \leq \frac{\delta}{4}\|\eta\|_{\mathcal{M}}^{2}+C \varepsilon^{2} E(0)^{p}\left\|u_{x}\right\|^{2} .
\end{aligned}
$$

At this point, choosing $\varepsilon>0$ such that

$$
C \varepsilon E(0)^{p} \leq \frac{\varkappa}{4}
$$

the inequality above turns into

$$
\varepsilon \int_{0}^{\infty} \rho(s)\left\langle B^{-1}\left(u^{p} u_{x}\right), A \eta(s)\right\rangle \mathrm{d} s \leq \frac{\delta}{4}\|\eta\|_{\mathcal{M}}^{2}+\frac{\varepsilon \varkappa}{4}\left\|u_{x}\right\|^{2} .
$$

Collecting (4.5)-(4.8), the proof is finished.

Remark 4.3. Observe that the constants $\alpha, \beta, \gamma$ can be explicitly calculated in terms of the structural quantities of the problem, even in an optimal way.

We are now in a position to prove Theorem 4.1. First we consider the energy identity (3.3) which, in light of (4.1), yields

$$
\frac{\mathrm{d}}{\mathrm{d} t} E+\frac{\delta}{2}\|\eta\|_{\mathcal{M}}^{2}+\frac{1}{2} \Gamma[\eta] \leq 0 \text {. }
$$

Next, setting

$$
\Lambda_{\varepsilon}(t)=E(t)+\Psi_{\varepsilon}(t)
$$

and taking the sum of (4.4) with the inequality above, we obtain the estimate (valid whenever $\varepsilon E(0)^{p} \leq \gamma$ )

$$
\frac{\mathrm{d}}{\mathrm{d} t} \Lambda_{\varepsilon}+\frac{\varepsilon \varkappa}{4}\left\|u_{x}\right\|^{2}+\frac{\delta}{4}\|\eta\|_{\mathcal{M}}^{2}+\left(\frac{1}{2}-\beta \varepsilon\right) \Gamma[\eta] \leq 0 .
$$

Due to (2.1) and (4.3), it is apparent to see that fixing ${ }^{1}$

$$
\varepsilon=\min \left\{\frac{1}{2 \alpha}, \frac{1}{2 \beta}, \frac{\delta}{\varkappa}, \frac{\gamma}{E(0)^{p}}\right\}
$$

and calling

$$
\varpi=\frac{\varkappa \lambda_{1}}{8\left(1+\lambda_{1}\right)}>0
$$

the inequality

$$
\frac{\mathrm{d}}{\mathrm{d} t} \Lambda_{\varepsilon}+\varpi \varepsilon \Lambda_{\varepsilon} \leq 0
$$

holds. Hence, applying the Gronwall lemma and (4.3) once more, we infer that

$$
E(t) \leq 4 E(0) \mathrm{e}^{-\varpi \varepsilon t}
$$

\footnotetext{
1 If $E(0)=0$ we can take any $\varepsilon$.
} 
We now set

$$
t_{0}=\frac{\log _{+}(4 E(0))}{\varpi \varepsilon} .
$$

Note that $t_{0}$, besides on $|I|, \mu, \alpha, \beta, \gamma$, depends also on $E(0)$ and the exponent $p$. However, it is clear that for every $t \geq t_{0}$

$$
E(t) \leq 1
$$

hence, by the semigroup property,

$$
E(t)=\left\|S(t) z_{0}\right\|_{\mathcal{H}}^{2}=\left\|S\left(t-t_{0}\right) S\left(t_{0}\right) z_{0}\right\|_{\mathcal{H}}^{2} \leq 4 \mathrm{e}^{\omega t_{0}} \mathrm{e}^{-\omega t}, \quad \forall t \geq t_{0}
$$

for some positive $\omega$, which now is independent of $p$ and $E(0)$. On the other hand, in light of (3.4),

$$
E(t) \leq E(0) \mathrm{e}^{\omega t_{0}} \mathrm{e}^{-\omega t}, \quad \forall t<t_{0} .
$$

In summary, defining

$$
\mathcal{Q}_{p}(E(0))=\max \{4, E(0)\} \mathrm{e}^{\omega t_{0}},
$$

the conclusion follows.

\section{Further remarks}

I. Up to minor modifications, it is possible to allow the presence of (even infinitely many) jumps in the memory kernel $\mu$. Indeed, denoting with $\left\{s_{n}\right\}_{n \geq 1}$ the increasing sequence of discontinuity points of $\mu$ and setting

$$
\mu_{n}=\mu\left(s_{n}^{-}\right)-\mu\left(s_{n}^{+}\right) \geq 0,
$$

we still have the energy identity (3.3) with

$$
\Gamma[\eta]=-\int_{0}^{\infty} \mu^{\prime}(s)\left\|\eta_{x}(s)\right\|^{2} \mathrm{~d} s+\sum_{n} \mu_{n}\left\|\eta_{x}\left(s_{n}\right)\right\|^{2} \geq 0 .
$$

In turn, the conclusions of Lemma 4.2 and Theorem 4.1 remain true (see e.g. $[23])$.

II. Condition (4.1) can be relaxed: Theorem 4.1 holds even if the kernel $\mu$ fulfills for some $C \geq 1$ and $\delta>0$ the weaker assumption

$$
\mu(t+s) \leq C \mathrm{e}^{-\delta t} \mu(s),
$$

for every $t \geq 0$ and almost every $s \in \mathbb{R}^{+}$, provided that $\mu$ is not too flat (cf. $[11,23])$.

III. In the linear case (i.e. when $p=0$ ) exponential decay can be shown within optimal assumptions on $\mu$, by means of linear techniques (see [25]). In this situation, besides (5.1), it is sufficient to assume that the kernel is not completely flat, namely, the set

$$
\mathfrak{D}=\left\{s \in \mathbb{R}^{+}: \mu^{\prime}(s)<0\right\}
$$

has positive Lebesgue measure. 


\section{References}

[1] Albert, J.: Dispersion of low-energy waves for the generalized Benjamin-BonaMahony equation. J. Differ. Equ. 63, 117-134 (1986)

[2] Albert, J.: On the decay of solutions of the generalized Benjamin-Bona-Mahony equation. J. Math. Anal. Appl. 141, 529-537 (1989)

[3] Amick, C.J., Bona, J.L., Schonbek, M.E.: Decay of solutions of some nonlinear wave equations. J. Differ. Equ. 81, 1-49 (1989)

[4] Benjamin, T., Bona, J., Mahony, J.: Models equations for long waves in nonlinear dispersive systems. Philos. Trans. R. Soc. Lond. Ser. A. 272, 47-78 (1972)

[5] Bona, J.L., Dougalis, V.A.: An initial and boundary-value problem for a model equation for propagation of long waves. J. Math. Anal. Appl. 75, 503-522 (1980)

[6] Byatt-Smith, J.G.B.: The effect of laminar viscosity on the solution of the undular bore. J. Fluid Mech. 48, 33-40 (1971)

[7] Chehab, J.P., Garnier, P., Mammeri, Y.: Long-time behavior of solutions of a BBM equation with generalized damping. arXiv:1402.5009v1 (2014) (24 pages)

[8] Chepyzhov, V.V., Pata, V.: Some remarks on stability of semigroups arising from linear viscoelasticity. Asymptot. Anal. 46, 251-273 (2006)

[9] Dafermos, C.M.: Asymptotic stability in viscoelasticity. Arch. Rational Mech. Anal. 37, 297-308 (1970)

[10] Dutykh, D.: Visco-potential free-surface flows and long wave modelling. Eur. J. Mech. B Fluids 28, 430-443 (2009)

[11] Gatti, S., Miranville, A., Pata, V., Zelik, S.: Attractors for semilinear equations of viscoelasticity with very low dissipation. Rocky Mt. J. Math. 38, 11171138 (2008)

[12] Ghidaglia, J.M.: Finite-dimensional behavior for weakly damped driven Schrödinger equations. Ann. Inst. H. Poincaré Anal. Non Linéaire 5, 365405 (1988)

[13] Giorgi, C., Naso, M.G., Pata, V.: Exponential stability in linear heat conduction with memory: a semigroup approach. Commun. Appl. Anal. 5, 121-133 (2001)

[14] Grasselli, M., Pata, V.: A reaction-diffusion equation with memory. Discrete Contin. Dyn. Syst. 15, 1079-1088 (2006)

[15] Guo, C., Fang, S.: Optimal decay rates of solutions for a multidimensional generalized Benjamin-Bona-Mahony equation. Nonlinear Anal. 75, 3385-3392 (2012)

[16] Gurtin, M.E., Pipkin, A.C.: A general theory of heat conduction with finite wave speeds. Arch. Rational Mech. Anal. 31, 113-126 (1968)

[17] Hayashi, N., Kaikina, E.I., Naumkin, P.I.: Large time asymptotics for the BBMBurgers equation. Ann. Henri Poincaré 8, 485-511 (2007) 
[18] Kakutani, T., Matsuuchi, K.: Effect of viscosity on long gravity waves. J. Phys. Soc. Jpn. 39, 237-246 (1975)

[19] Korteweg, D.J., Vries, G.de : On the change of form of long waves advancing in a rectangular canal and on a new type of long stationnary waves. Philos. Mag. 39, 422-443 (1895)

[20] Larkin, N.A., Vishnevskii, M.P.: Decay of the energy for the Benjamin-BonaMahony equation posed on bounded intervals and on a half-line. Math. Methods Appl. Sci. 35, 693-703 (2012)

[21] Mammeri, Y.: Comparaison entre modèles d'ondes de surface en dimension 2. M2AN Math. Model. Numer. Anal. 41, 513-542 (2007)

[22] Miranville, A., Temam, R.: Mathematical Modelling in Continuum Mechanics. Cambridge University Press, Cambridge (2001)

[23] Pata, V.: Exponential stability in linear viscoelasticity. Q. Appl. Math. 64, 499$513(2006)$

[24] Pata, V.: Stability and exponential stability in linear viscoelasticity. Milan J. Math. 77, 333-360 (2009)

[25] Pata, V.: Exponential stability in linear viscoelasticity with almost flat memory kernels. Commun. Pure Appl. Anal. 9, 721-730 (2010)

[26] Peter, J.G., Gurtin, M.E.: On the theory of heat condition involving two temperatures. Z. Angew. Math. Phys. 19, 614-627 (1968)

[27] Stanislavova, M.: On the global attractor for the damped Benjamin-BonaMahony equation. Discrete Contin. Dyn. Syst. suppl, 824-832 (2005)

[28] Temam, R.: Infinite-Dimensional Dynamical Systems in Mechanics and Physics. Springer, New York (1997)

[29] Wang, B.: Strong attractors for the Benjamin-Bona-Mahony equation. Appl. Math. Lett. 10, 23-28 (1997)

[30] Wang, M.: Long time dynamics for a damped Benjamin-Bona-Mahony equation in low regularity spaces. Nonlinear Anal. 105, 134-144 (2014)

[31] Wang, B., Yang, W.: Finite-dimensional behaviour for the Benjamin-BonaMahony equation. J. Phys. A Math. Gen. 30, 4877-4885 (1997)

Filippo Dell'Oro

Institute of Mathematics of the Academy of Sciences of the Czech Republic

Žitná 2511567 Prague 1

Czech Republic

e-mail: delloro@math.cas.cz 
Youcef Mammeri

Laboratoire Amiénois de Mathématique Fondamentale et Appliquée CNRS UMR 7352

Université de Picardie Jules Verne

80069 Amiens, France

e-mail: youcef.mammeri@u-picardie.fr

Vittorino Pata

Dipartimento di Matematica "F. Brioschi"

Politecnico di Milano

Via Bonardi 9

20133 Milan

Italy

e-mail: vittorino.pata@polimi.it

Received: 7 November 2014.

Accepted: 12 December 2014. 